\title{
New cardiomyocyte formation in adult mouse hearts
}

\section{ce}

new

cardiomyocytes

can be

generated in

the adult heart

from pre-

existing adult cardiomyocytes

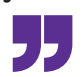

Previous studies have shown that new cardiomyocytes can be generated in the adult mammalian heart, but the source of these new cardiomyocytes was unclear. In a new study published in Circulation, Zeng and colleagues report that existing adult cardiomyocytes can re-enter the cell cycle and form new cardiomyocytes via a three-step process involving dedifferentiation, proliferation, and redifferentiation.

"Myocyte proliferation is generally infrequent and thus difficult to study," explains Chunyu Zeng,

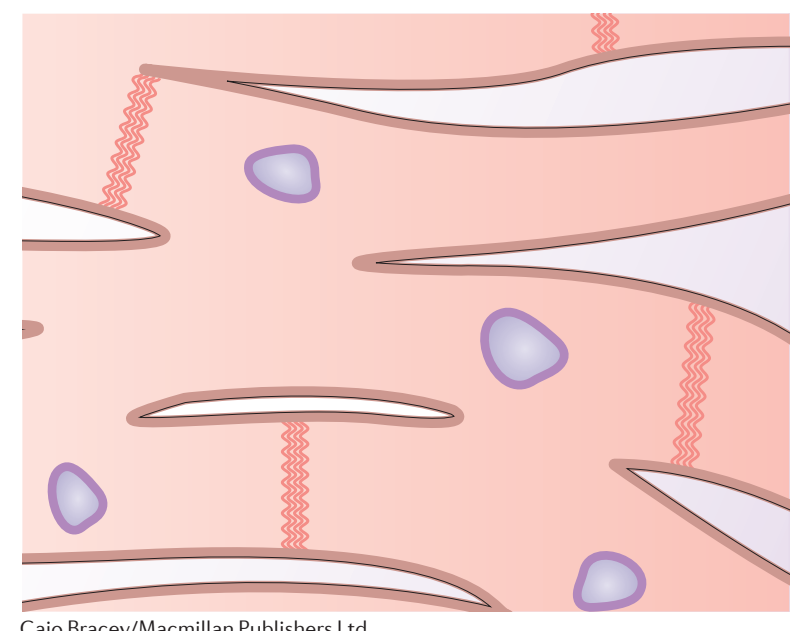

Caio Bracey/Macmillan Publishers Ltd senior investigator of the study. "As such, we developed an experimental system to induce new cardiomyocyte formation from adult cardiomyocytes, and used this system to explore the underlying mechanisms of new cardiomyocyte formation." Genetically labelled cardiomyocytes derived from adult mouse hearts were cultured with neonatal rat ventricular myocytes and assessed via time-lapse imaging and immunostaining to observe the cytokinesis of proliferating adult cardiomyocytes. Adult cardiomyocytes in co-culture stained positive for several proliferation markers, suggesting that those cells had re-entered the cell cycle, in a process of cardiomyocyte dedifferentiation. These dedifferentiated adult cardiomyocytes developed an organized contractile apparatus by day 3 , and reached a peak number of beating cardiomyocytes by day 7 . This in vitro system showed that the process of myocyte uncoupling from neighbouring cardiomyocytes, dedifferentiation and proliferation of uncoupled myocytes, and redifferentiation of these cells into functional cardiomyocytes are involved in new cardiomyocyte formation from pre-existing adult cardiomyocytes. Both mononucleated and bi/multinucleated adult cardiomyocytes were shown to proliferate with completed cytokinesis. Subsequent in vivo studies in mouse models of myocardial infarction showed that enhancing electrical coupling between adult cardiomyocytes in the infarct border zone can increase the number of newly formed cardiomyocytes and improve cardiac function.

"Our study confirmed that new cardiomyocytes can be generated in the adult heart from pre-existing adult cardiomyocytes," concludes Zeng. "These novel findings might create new approaches to enhance the dedifferentiation-proliferationredifferentiation process of adult cardiomyocytes in vivo, which would be a significant step in cardiac regeneration for damaged hearts and helpful for patients with myocardial infarction and heart failure."

Karina Huynh

ORIGINAL ARTICLE Wang, W. E. et al.

Dedifferentiation, proliferation and redifferentiation of adult mammalian cardiomyocytes after ischemic injury. Circulation https://doi.org/10.1161/ CIRCULATIONAHA.116.024307 (2017) 